\title{
A BRIEF SYNTHESIS ON COMPLEX SYSTEMS
}

\author{
Uthamacumaran, A. \\ a_utham@live.concordia.ca \\ Concordia University, Montreal.
}

What is a complex system? The definition of a complex system remains somewhat ambiguous. A complex system can be defined as a system consisting of many interacting parts exhibiting emergent behaviors. The emerging field of complexity science entails a change in the language of scientific research and thinking. As such, the general properties, tools, and definitions pertaining to complex systems must be made accessible in layman terms to multi-disciplinary systems scientists and thinkers. With this intention, this literary survey presents the development and glossary of essential concepts steering complex systems.

Keywords: Complex Systems; Emergence; Complexity; Chaos; Fractals; Networks 


\section{INTRODUCTION TO COMPLEXITY}

Complex systems theory, or simply complexity science is the interdisciplinary field of systems science which attempts to provide a wholistic description of complex systems through the (distortion) lens of information, as opposed to the (traditional) reductionist approaches [1]. Complex systems are systems composed of many nonlinearly and locally interacting parts (agents) which give rise to emergent behaviors [2]. However, the threshold for 'many' is subjected to debate depending on the system and discipline of science to which one adheres. Some general properties of complex systems include selforganization, adaptation, pattern formation, criticality, dynamic multi-scale structures, multi-nested feedback loops, nonequilibrium processes and nonlinearity $[3,4]$. In simple terms, a complex system is a system in which the collective whole is more than the sum of its interacting parts. The description of complex systems requires a new set of tools and language to communicate in between broad disciplines of investigators. Such is the motivation for this brief layman survey.

The definition of complexity may vary from one discipline of science to another. Complexity theory is often confused with computational science, wherein computational complexity theory deals with the study of time, space and algorithmic resources required by computers to efficiently perform a task [5]. There are then descriptive measures of complexity such as algorithmic complexity, or Kolmogorov complexity which denotes the shortest string of bits required to effectively compute an algorithm $[6,7]$. Such measures are of importance in network biology, where they quantify to some approximation the network complexity in terms of its information content [8]. However, the review will not focus on such branches of (computational) complexity and strictly focus on the more general complex systems. To make this illusory distinction, the term complex systems will be used mostly instead of complexity.

\section{THE ORIGIN OF COMPLEX SYSTEMS}

According to Merriam-Webster's dictionary, the word complexity takes root in the latin word complectere or complecti, meaning to entwine or to infold. It is a compound of com- "together" and plectere "to weave, or to braid". Complex thus means composed of interconnected and interacting parts. Complex systems are then systems in which the whole is composed of interrelated parts. Complex systems are thus whole systems.

The details of when and where complex systems surged as a science is debatable depending on the disciplines of science. Most complexity scientists would accept the 1960s with the emergence of dynamical systems theory, or the 1980s when complex systems theory was being developed as a branch of systems science. In 1963, Edward Lorenz demonstrated the first numerical model of a strange attractor, the Lorenz attractor, by studying three coupled ordinary differential equations in attempt to describe weather turbulence [9]. This was a revolution which initiated the formal study of chaos theory and nonlinear dynamics. In the 1970s, Mandelbrot realized natural systems whether it be financial stock markets, the roughness of terrestrial landscapes, social networks or biological structures can be described using a descriptive statistical measure - the fractal dimension [10]. That is the stratification of these systems have 'fractional' dimensions, a value in between two consecutive integers. Nonlinear dynamics, fractals and chaos remain the essence of complex systems. Fractals have been extended into the study of complex systems such as cancer biology where it is generally believed cancers exhibit higher fractal dimensions $[11,12]$. It was also at this time, the early 70 s when Ruelle and Takens coined the term strange attractor to describe the multi-fractal patterns observed in fluid turbulence [13]. To most complexity thinkers, turbulence is the ideal picture of a complex system. Despite its universality, finding the solutions to the Navier-Stokes Equations governing fluid turbulence, remains the holy grail of 
mathematical physics. The major developments in the physics of fluids, their chaotic pattern formations and the strange attractors emerging in their flows are hot topics in complex systems research [14].

To systems biologists, the origins of complex systems may date back to Lamarck and Darwin. Complex systems are essentially inspired by the complex patterns and structures formed by living systems. However, most systems biologists would agree network biology and hence, network science as the initial grounds to complexity science. In 1942, Waddington derived a metaphorical description of developmental biology, where cell fates are visualized as attractors rolling down valleys and hills on the epigenetic landscape [15]. Reconstructing the Waddington landscape as the phase-space dynamics of biological networks remains one of the central interests in systems biologists. In 1969, Kauffman derived the NK automaton, a Random Boolean Network model where genes can occupy a binary state of 0 or 1 representing on/off states [16]. Boolean networks are now universal tools in network biology. The physical foundations of network complexity were then established by mathematical physicists such as Barabasi and Newman $[17,18]$. Network science is now at the heart of complex systems, used to assess the structure-to-function relationships in complex systems such as genes and protein interactions [19].

It was Nobel laureate and physicist Murray Gell-man who co-found the Santa Fe institute for complexity science in 1984. Thus, complexity was institutionalized upon the pioneering works of a thinker in abstract particle physics. This is to no surprise since group theory and field theory study how complex structures emerge from higher-dimensional abstract algebra. Yet, to most physicists, complex systems may predate to Ludwig Boltzmann who set the foundations of statistical mechanics [20]. The laws of thermodynamics remain unrefutably the central dogma of complex systems. The free energy principle and its trade-off with entropy governs emergence [20]. Most physicists would argue complex systems takes foundations in statistical mechanics and later Shannon's information theory [21]. The shift in studying systems from the lens of energy towards that of information is central to the framework of complex systems. Complexity scientists are interested in how information flows and organizes into structures. However, this too is only a part of the skeleton of complexity.

The study of information flow and steering them in control systems roots to the transdisciplinary science of cybernetics [22]. Cybernetics was also amidst the initial conditions to Artificial Intelligence and manmade self-regulating systems. Quantifying information flow (dynamics) in complex networks remains a fundamental problem in systems science. Many statistical approaches in the study of timeseries information systems have been paved by Wiener [22] and remain to be deciphered in their application to complex systems/networks. To get a feeling for cybernetic systems, allow me to illustrate a few examples. Let us consider the cybernetics (information flows) of social networks.

Social networks such as those pertaining to human interactions (relationships) and societal structures are cybernetic systems; systems which can be visualized as a graph theoretic network with feedback loops controlling (regulating) their associative relationships. Now, think of Adolf Hitler, how did he succeed as a dictator of the masses? He was a positive feedback loop for the mentally unstable society at the devastating war- time, amplifying their collective behaviors towards synchronization by the transmission of certain information. Although this hub of supporters was a negative feedback system for society (as a whole), it was a positive feedback loop for Hitler. The supporters acted as coupled oscillators which kept preferentially supporting him in an amplifying manner (i.e., resonance). This collective oscillations-like description of complex networks applies to economic feedback systems as well, e.g., the rich get richer, the poor get poorer (i.e., the Pareto's principle). We refer to such networks as critical networks, due to their power law behaviors. Likewise, the same problem of criticality occurs in cancer gene expression - certain mutated gene networks form multi-nested feedback loops 
amplifying the signal of their associated-hubs to make cancer cells uncontrollably grow and invade, while suppressing the cries and calls of all other genes. Although the tumor-disfavoring networks seemingly form the majority, their less-densely connected structures and resultant outcries are not strong enough to inhibit the cascading effects of cancer-stemness networks. Therefore, the cybernetics (information dynamics) of tumor ecosystems is essentially a graph theory problem. Cybernetics is then the science of communication and feedback loops in these complex graph networks. Cybernetics poses the central question: How can certain nodes (e.g., people, cells, or genes) of a complex network control the collective dynamics of the whole system?

Then, there is the problem of defining information itself. There are different measures of quantifying information, namely, Shannon's information entropy, von Neuman's entropy, Kolmogorov's entropy, Fisher information, etc. [21]. Each measure describes information within a different study system. Contemporary physicists would rather argue complex systems is a spin-off of condensed (soft) matter systems. Perhaps this is because most soft matter systems (e.g., biosystems) are driven by nonequilibrium statistical mechanics and nonlinear dynamics, the bread and butter of complexity. Furthermore, condensed matter systems deal with many-body systems, wherein the interactions amidst larger degrees of freedom may result in complex, emergent behaviors [23]. Soft matter physics demonstrates nonlinear interactions between the many parts of a system give rise to collective, unexpected behaviors [23]. For example, active turbulence in dense suspensions of microtubules is a beautiful example to visualize complex pattern formations [24]. The hydrodynamical patterns are best described by variants of the Navier-stokes equations and not the conventional diffusion equations used to explain most biochemical systems suspended in a fluid [25].

In the 1980s, physicist David Bohm described quantum mechanics as a tool to investigate physics in the perspective of systems science [26]. He coined the term implicate and explicate order to describe the enfoldment and unfoldment (emergence) of information and structures/patterns from a whole system. The whole, he calls an undivided whole composed of fragments, where the many is subjected to the observer or type of explicate order [26]. He provides the analogy of how the information carried to an antenna on an old television box is fine-tuned to a certain frequency at which the visuals and sound are observed on the TV. The observations are the unfolded (explicate) orders, while the information carried off as electromagnetic waves is the implicate order [26]. Such an analogy well suits our study of complex systems and how orders/patterns emerge within them at different levels of observation. Quantum mechanics, although not seen as an obvious part of complex systems research, shares many of the features and insights required to approach complex systems.

A computer scientist would argue the late 1930s when Church and Turing were laying the foundations of computation theory would be a better fit to the origin of complexity, although it pertains to computational complexity and not necessarily complex systems [5]. In fact, it was Alan Turing in 1952 who devised a mathematical model of chemical morphogenesis, that nonlinear, partial differential equations characterizing reaction-diffusion systems may describe the pattern formations observed in biological organisms [27]. Pattern formation is a central attribute of complex systems. These works were then extended into the domain of nonequilibrium statistical mechanics by Nobel Laureate and Chemist Prigogine. Prigogine demonstrated nonequilibrium chemical systems can spontaneously give rise to highly ordered, dissipative structures (i.e., order out of chaos) [28]. These works are amidst the primary grounds to the concepts of emergence and self-organization in complex systems. It must also be noted that the term order is then ambiguous since chaos itself is a type of complex order. The terms regular, predictable, or well-behaved may be better suited to characterize 'ordered' systems in contrast to chaotic systems. 
Today, computational simulations, machine learning and artificial intelligence are the state-of-the-art approaches in studying complex systems. Machine learning methods such as Deep learning neural networks are capable of complex pattern recognition from large complex datasets [29]. As such, inarguably computational physics is the contemporary foundations of complex systems science. It provides an alternate approach to investigating complex systems, whose behaviors tend to be nonlinear, adaptive and often chaotic [30]. As such, finding equations that well-model complex systems remain inadequate and if equations are found, finding analytical solutions to them remains intractable in most complex systems [30]. Hence, computational algorithms provide searching heuristics and approximation tools to model complex systems.

To the mathematicians, Kolmogorov made pioneering contributions to all of the above-defined branches of complexity. Kolmogorov's works on fluid turbulence remain the most coherent and well-developed mathematical bases in the understanding of the complex systems [31]. Kolmogorov's works demonstrate turbulent flows obey a power-law decay within its inertial range, wherein the eddies and vortex structures breakdown into a fractal hierarchy [31]. Kolmogorov also pioneered algorithmic information theory, a branch of computational complexity which studies the algorithms and resources required to solve a computational problem [5]. As mentioned, the K-complexity, also known as Kolmogorov or algorithmic complexity quantifies the shortest bits of a string or computer program required to describe a complex system. K-complexity is a robust measure of a network's complexity vastly unutilized in current approaches to network biology [5]. Kolmogorov also contributed to the study of biological morphogenesis and pattern formation (i.e., the FKPP equation) [32]. This brings into attention the Kuhnian model of science: how the various disciplines of science, and hence complexity, are driven by the socio-political history of science.

To socioeconomic scientists, perhaps complex systems goes back to the founding principles of Game theory, and to psychology scholars it may date back to the abstract teachings of Carl Jung who advocated the principles of wholeness in the study of consciousness [33, 34]. Jung often used the word complex to describe the connected group of repressed ideas within an individual or rather the connection between a person and the surroundings [34]. Thus, complexity even in the psycho-social sciences is related to the interconnectivity of a system with its surroundings. To scholars in the arts, complex systems may pertain to pieces of art encompassing creativity. How does one quantify creativity to the information content in literature, a painting, or a sonata? Studies on harmonic analysis, frequency distributions (e.g., Zipf's distribution) and statistical behaviors of artistic structures (e.g., multifractality) have been used as measures of creativity and hence, the emergence of complexity in artworks [35, 36]. However, most artists will simply seek to describe creative works whether it be Carroll's Alice in Wonderland, Mozart's symphonies or the swirly flows of Van Gogh's starry night as complex systems that cannot be defined using words from human language. Artistic creativity can only be appreciated for its beauty and not analyzed (reduced to its parts). Such sentiments are to be shared in the descriptions of real-world, complex systems.

While the time-point one may choose to better represent their personalized perspective of complexity may vary, complex systems are universal and are signatures of Nature. All the tools and disciplines described above cohere into a single framework in complex systems. It is an interdisciplinary science which cannot be reduced to parts, and as such cannot be separated to specific branches of science. To illustrate, consider the following example. Network biology is now being investigated within the framework of algorithmic complexity theory. In the emerging field of Algorithmic Information Dynamics 
(AID), biological networks are investigated as dynamical systems in software space using perturbation analysis [37]. Measures of algorithmic information content are then approximated using algorithms such as Block Decomposition Method or lossless compression algorithms to quantify the network complexity [37]. This is a clear example of how interconnected various traditional disciplines of science have become in the study of complex systems. The AID framework combines tools from dynamical systems theory, computational physics, network science and biology into a whole systems science.

\section{THE FUNDAMENTALS OF COMPLEX SYSTEMS}

The following concepts and tools are the meat on the bones (substance) of complexity science. They are general characteristics and tools that apply to investigating any type of complex system, and hence should be appreciated by systems researchers. Note however nonequilibrium statistical mechanics, nonlinear dynamics and chaos are not enlisted here for they are not simple concepts. They are the foundations of complexity science and cannot be confined to this layman survey.

Emergence: The current definition of a complex system implies emergence- the appearance of properties, patterns, and behaviors in a system, due to nonlinear interactions, not foreseen within the parts of the system [38]. For example, consider the flight of a bird or the swimming of a fish. The flow patterns of species across ecosystems exhibits emergent structures. As a single agent, the bird's flight or the trajectory of a fish may seem apparently random but driven by its own survival instincts, the search for food, shelter, light, and resources. However, collectively groups of birds and schools of fishes exhibit organizational adaptability and emergent behaviors that are unforeseen by the individuals [38, 39]. The flocking of birds creates complex patterns following the Vicsek model, where birds follow a simple rule of behavior: stay close to your neighbors but not too close. However, collectively the flocking patterns appear to behave like hydrodynamic fluids [39]. The same description applies to the schools of fishes. Their collective motion displays a continuum model resembling fluid dynamics [39].

Thus, complex (adaptive) systems show systems following simple rules can exhibit unpredictable patterns and collective behaviors. Emergence is not necessarily observed in natural systems. For example, computational models such as Conway's Game of Life and elementary Cellular Automata show that simple computer programs following local, discrete rules exhibit emergent behaviors resembling those observed in natural systems [40]. These computational models also show complex systems often exhibit computational irreducibility, intractability, undecidability, and unpredictability [40]. Such notions cannot be defined by studying a complex system in terms of differential equations.

Self-organization: Self-organization (SO) refers to the spontaneous order that arises from local interactions between the parts of an initially disordered system, without the need for any external mediators [41]. The above-discussed emergent behaviors may be described as self-organized processes as well. It must be noted that order can also be ambiguous. For example, Prigogine describes chaos as a form of order in which the temporal sequence is complex. However, herein order will be defined as a system in which entropy is low. In 1944, physicist Erwin Schrodinger described the emergence of life as a nonequilibrium thermodynamic process driven by negentropy (i.e., the system lowers its own entropy while increasing the entropy of its surroundings) [42]. These principles were accounted by Schrodinger to describe the spontaneous order of genetic information, 'aperiodic crystals', which today is known as the chromatin [42]. Crystal formation, the arrangement of initially disordered molecules into highly structured lattices is thus a clear example of self-organization [23]. The ordered arrangement of chromatin in the metaphase plate during cellular division, driven by various locally interacting protein complexes such as microtubules and cell polarity complexes is a biological example of SO [43]. SO is 
inherently linked to regularity and symmetry. We see the consequences of perturbed/aberrant SO in the asymmetric chromosomal segregation in pathological systems such as cancer cells. Another example of self-organization is stigmergy. Consider ant colonies, where individually an ant's behavior may seem to be of very little intelligence and behave like a random walker [39]. However, collectively, the swarming of ant colonies exhibit intelligent structures and highly coordinated task performances [39]. Thus, selforganization is a feature of most complex adaptive systems.

Phase-transition and Criticality: Most complex systems display critical behaviors. That is, the system is at the tipping point between chaos and order [44]. The ant colonies defined in SO may also be described as a system of Self-organized Criticality (SOC). Kauffman describes healthy cellular phenotypes as critical attractors of the biological network's state space (i.e., edge of chaos) [16]. The cell membrane may be defined as a critical structure poised between a solid and a fluid (i.e., fluid-mosaic model). Thus, criticality denotes the point at which a system may display a phase-transition. A phase-transition occurs when a system undergoes symmetry-breaking, i.e., when small fluctuations acting on a system crossing a critical point decides the system's fate [45]. In complex systems such as fluid turbulence and the phase-space portrait of any chaotic system, a phase transition is denoted by the bifurcation of a system at that critical point [46]. For example, above a certain critical threshold of growth parameters, the logistic model will exhibit chaotic behaviors (i.e., period-doubling bifurcations) [46]. The Feigenbaum constant and fractal measures are then used to characterize the complex behaviors and structures emerging in these chaotic systems. It is self-evident that for one to truly understand complex systems, an appreciation of dynamical systems theory is a fundamental pre-requisite.

Networks: Networks are one of the most widely used tools in the study of complex systems. Network science was found upon the disciplines of graph theory and information theory. Graph theory was developed by Swiss Mathematician Leonhard Euler. It is the branch of mathematical sciences which studies networks, abstract structures used to symbolize the pairwise relationships between objects. The graph network is then a visual representation of the associations amidst the various elements of the network, where the elements (e.g., proteins, genes, etc.) are represented as nodes (vertices) of the graph and their interactions by links (edges) $[17,18]$. Complex systems form complex networks, with multi-level, multi-scale structures and nonlinear interactions [47].

The measures of a network's complexity may pertain to the description of its structure and dynamics. Structural features of a network include the degree, distance, betweenness, and clustering coefficient while the dynamic features of a network are more complex to define and can only be inferred from statistical tools such as entropy, mutual information, algorithmic complexity, etc. (i.e., collectively referred to as information-graph theoretic methods) $[19,47]$. For example, the gene interaction networks (GRN) and protein-protein interaction (PPI) of cancers are highly dynamic, complex networks [19]. The high-dimensional phase-space projection of these networks forms the epigenetic/attractor landscape, where the cellular phenotypes may correspond to attractors of the landscape [48]. An attractor is visualized as a valley (local energy minimum) of this state-space landscape. Attractors are abstract mathematical structures derived from dynamical systems theory which may be of many types: fixed-points, stable equilibria, periodic oscillators, limit cycles, critical, and chaotic (strange) [46]. Growing evidence suggest that cancer cells are unstable, aperiodic attractors of their network state space exhibiting nonequilibrium statistical mechanics (i.e., fluctuations around an attractor/valley) [49]. Whether these unstable attractors may be defined as strange attractors or not remains a poorly investigated topic in network biology [37]. 
Adaptation: The term does not deviate from that defined in Darwin's Origin of Species. Complex adaptive systems (CAS) are a category of complex systems which respond to the perturbations from external and/or internal environment by changes that allow the selection of their population's survival fitness [50]. For example, cancer cells are CAS. There need not be more said about adaptation, but it is critical to consider that even in simple biosystems, adaptation is difficult to predict and characterize. There are many layers of complexity in biological networks, which makes comprehending CAS a complicated process $[19,50]$. The complex networks in CAS tend to form multi-nested networks with dynamic feedback loops. Hence, the study of adaptation requires principles of control systems and cybernetics (i.e., the systems science of information processing) [22].

Pattern Formation: Pattern formation is inseparable from the above-discussed aspects of a complex system. Think of clouds, morphogenesis, bifurcating structures in plants and trees, etc. Herein, I draw attention to a sub-class of pattern formation observed in natural systems- fractals and multifractals, where the latter denotes fractals with dynamic scaling exponents [10]. Fractals are ubiquitously observed in nature, yet they do not have any closed-form analytical solutions [10]. Most chaotic and nonequilibrium systems exhibit multifractal structures, such as the strange attractors in fluid turbulence, the blood vessels networks in tumor angiogenesis, and the fluctuations of stock markets [14, 51]. Multifractal analysis is emerging as a tool in tumor texture characterization and diagnosis [52].

Computation and Algorithms: These are perhaps the most powerful tools in current complex systems research. An algorithm is a recipe or a set of instructions to perform a task. In many ways it is difficult to distinguish an algorithm from a computer, but for simplicity we may take a computer to be a system which can integrate and execute algorithm(s) [5]. Artificial Intelligence (AI), machine learning and data mining are used to recognize patterns and quantify information flow in complex systems. For example, Deep Learning is emerging as the frontier of precision oncology and is a powerful tool for causal structure inference in cancer networks [53]. This survey is not intended to educate on the various tools and approaches available in computational sciences. However, it must be noted that although Al algorithms can provide predictions of certain patterns and behaviors in complex systems, they do not necessarily provide an understanding of the whole system [54]. They are to be treated as tools to gain insights into what may constitute the whole or well-model the whole and not the whole itself [54]. The same implication applies to all other tools of complexity discussed above. Regardless, simulations, computational modelling and $\mathrm{Al}$-applications are amidst the most promising prospects to complex systems research. Furthermore, there are tools such as agents-based modelling, evolutionary algorithms, etc. not discussed herein that falls into computational methods for complex systems science [1].

\section{CONCLUSION}

Complex systems theory is a paradigm shift in systems thinking and research, wherein the key concepts required to appreciate the emerging science are briefly described above. The survey is written to stimulate systems thinkers towards adopting complexity science and its tools to approach real-world problems. Therefore, it is essential the thinkers from broad disciplines of investigation integrate as a whole, alike a complex system, to make true progress in finding solutions to these real-world problems. As Quantum Mechanics demonstrates, the observer, the measurement (observation) and that which is observed (the system) are intertwined and should not be separated [26]. That is, humans and the science driven by them are complex systems.

DECLARATION STATEMENT: There are no competing interests. I am the sole author of this article. 


\section{REFERENCES}

1. Mitchell, M. Complexity: A guided tour (Oxford University Press, 2009).

2. Bossomaier, T.R.J. and Green, D.J. Complex Systems. Cambridge Univ. Press, 2000

3. Cross, M. and Greenside H., Pattern Formation and Dynamics in nonequilibrium systems. Cambridge University Press. (2009)

4. Newman, M.E.J., Resource Letter CS-1: Complex Systems. Am. J. Phys. 79, 800 (2011)

5. Sipser, M. Introduction to the Theory of Computation. (Thomson, Boston, MA, 2nd edition, 2006).

6. Shalizi, C.R. "Methods and techniques of complex systems science: An overview." In Complex systems science in biomedicine, pp. 33-114. Springer, Boston, MA, (2006).

7. Lloyd, S. Measures of complexity: a non-exhaustive list. IEEE Control Systems Magazine 21(4):7-8 (2001)

8. Zenil, H., Kiani, N. A., and Tegner, J., A review of graph and network complexity from an algorithmic information perspective. Entropy. 20, 551 (2018)

9. Lorenz, E.N. Deterministic nonperiodic flow. J. Atm. Sci. 20:130-141 (1963)

10. Mandelbrot, B. The Fractal Geometry of Nature (W. H. Freeman, New York, 1983)

11. Coffey, D.S. Self-organization, complexity and chaos: The new biology for medicine. Nat Med. 4(8): 882-885 (1998)

12. Baish JW, Jain RK. Fractals and cancer. Cancer Res. 60(14):3683-8 (2000)

13. Ruelle, D. Turbulence, strange attractors and chaos. World Scientific Ser A. 16. (1995)

14. Sreenivasan, K.R. and Meneveau, C. The multifractal nature of turbulent energy dissipation. J. Fluid Mech. Vol 224: 429-484 (1991)

15. Waddington, C.H. The Canalization of development and the inheritance of acquired characters. Nature 150, 563-565 (1942)

16. Kauffman, S.A. At Home in the Universe (Oxford University Press, Oxford, 1995).

17. Barabasi, Albert-Laszlo. Network Science, Cambridge University Press. (2016)

18. Newman, M. E. J.: The Structure and Function of Complex Networks. SIAM Rev. 45(2):167-256, 2003. 
19. Barabasi, A-L and Oltvai, Z., Network biology: understanding the cell's functional organization. 5. 101-113 (2014)

20. Sethna, J. P., Statistical Mechanics: Entropy, Order Parameters and Complexity (Oxford University Press, Oxford, 2006).

21. Cover, T.M. and Thomas, J.A., Elements of Information Theory (John Wiley, New York, 1991).

22. Wiener, N. Cybernetics: Or control and communication in the animal and the machine ( $2^{\text {nd }}$ version. MIT Press, 1961).

23. Doi, M. Soft matter physics (Oxford University Press, 2013)

24. Wensink HH, Dunkel J, Heidenreich S, Drescher K, Goldstein RE, Löwen H, Yeomans JM. Mesoscale turbulence in living fluids. PNAS, 109(36): 14308-13 (2012)

25. Huber, L., Suzuki, R., Kruger, T., Frey, E., \& Bausch, A.R. Emergence of coexisting ordered states in active matter systems. Science. 361(6399): 255-258 (2018)

26. Bohm, D. Wholeness and the Implicate Order (Routledge, 1980).

27. Turing, A.M. The chemical basis of morphogenesis. Philos. Trans. R. Soc. Lond. B: Biol. Sci. 237 (641): 37-72 (1952)

28. Prigogine, I., Stengers, I. Order out of Chaos: Man's new dialogue with nature (Flamingo, 1984)

29. LeCun, Y. et al., Deep Learning. Nature. volume 521: 436-444(2015)

30. Wolfram, S. Complex Systems Theory. In Emerging Syntheses in Science: Proceedings of the Founding Workshops of the Santa Fe Institute. [Addison-Wesley Ed.] pp. 183-189 (1988)

31. Kolmogorov, A. The local structure of turbulence in incompressible viscous fluid for very large Reynolds numbers. Cr Acad. Sci. URSS 30, 301-305, 1941

32. Kolmogorov, A., Petrovskii, I. \& Piskunov, N. Study of the diffusion equation with growth of the quantity of matter and its application to a biological problem. Bull. State Univ. Mos., 1-25 (1937)

33. Nash, J. (1950) "Equilibrium points in n-person games" PNAS. 36(1):48-49.

34. Jung, C.G. Synchronicity: An acausal connecting principle (Princeton University Press, 1960)

35. Powers, D. M.W (1998). Applications and explanations of Zipf's law. Association for Computational Linguistics: 151-160.

36. Ball, P. Van Gogh painted perfect turbulence. Nature News (2006) doi:10.1038/news060703-17 
37. Zenil, H. et al., An algorithmic information calculus for causal discovery and reprogramming systems. iScience. 19, 1160-1172 (2019)

38. Strogatz, S. Sync: The Emerging Science of Spontaneous Order. (Hyperion, New York, 2003).

39. Ladyman, J. and Wiesner, K. "What is a complex system?". Yale University Press (2020).

40. Wolfram, S. A New Kind of Science. Wolfram Media (2002)

41. Gros, C. Complex and adaptive dynamical systems: A primer. Springer-Verlag, 2nd edition (2011)

42. Schrodinger, E. What is life? (Cambridge University Press, 1944)

43. Heald, R. et al., Self-organization of microtubules and motors. Nature volume 389, pages305308 (1997)

44. Zimelis, K. Complex systems. Nature 410, 241 (2001)

45. Domb, C. Phase transitions and critical phenomena (Academic Press, 1972)

46. Strogatz, S.H. Nonlinear Dynamics and Chaos: With Applications to Physics, Biology, Chemistry, and Engineering (Studies in Nonlinearity). (2nd Ed.) (CRC Press, 2015)

47. Harush, U. and Barzel, B. Dynamic patterns of information flow in complex networks. Nature Communications volume 8: 2181 (2017)

48. Huang, S. Genomics, Complexity and drug discovery: insights from Boolean network models of cellular regulation. Pharmacogenomics, 2, 203-222 (2001)

49. Huang, S. and Kauffman, S. 2013. How to escape the cancer attractor: rationale and limitations of multi-target drugs. Semin Cancer Biol. 23(4): 270-278

50. Miller, J.H. and Page, S.E., Complex Adaptive Systems (Princeton University Press, Princeton, 2007).

51. Baish JW, Jain RK. Cancer, Angiogenesis and fractals. Nat Med. 4(9): 984 (1998)

52. Islam, A., et al., Multifractal texture estimation for detection and segmentation of brain tumors. IEEE Trans Biomed Eng. 60(11): 3204-3215 (2013)

53. Topol, E.J. High Performance medicine: the convergence of human and artificial intelligence. Nat Med. 25, 44-56 (2019)

54. Bishop, C.M. Pattern Recognition and Machine Learning (Springer, 2006) 